ÉGYPTE monde arabe

\section{Égypte/Monde arabe}

$21 \mid 1995$

Économie égyptienne et perspectives de paix au Proche-Orient

\title{
Quelques caractéristiques de l'évolution économique de l'Égypte depuis 1991
}

\section{Fathi Ibrahim}

\section{(2) OpenEdition}

\section{Journals}

Édition électronique

URL : https://journals.openedition.org/ema/367

DOI : $10.4000 /$ ema.367

ISSN : 2090-7273

Éditeur

CEDEJ - Centre d'études et de documentation économiques juridiques et sociales

Édition imprimée

Date de publication : 31 mars 1995

Pagination : 11-18

ISSN : 1110-5097

\section{Référence électronique}

Fathi Ibrahim, «Quelques caractéristiques de l'évolution économique de l'Égypte depuis 1991 », Égypte/Monde arabe [En ligne], 21 | 1995, mis en ligne le 08 juillet 2008, consulté le 07 juillet 2022 URL : http://journals.openedition.org/ema/367 ; DOI : https://doi.org/10.4000/ema.367

Ce document a été généré automatiquement le 7 juillet 2022

Tous droits réservés 


\title{
Quelques caractéristiques de l'évolution économique de l'Égypte depuis 1991
}

\author{
Fathi Ibrahim
}

1 L'importance des facteurs extérieurs dans l'évolution de l'Égypte contemporaine se mesure bien à l'aune de sa dépendance économique. Après avoir connu un taux de croissance réelle du PIB de 9\% par an entre 1974 et 1984, pour des raisons essentiellement externes, l'économie égyptienne a stagné en dépit d'une aide principalement américaine - massive, et a abordé la décennie 1990 en état de banqueroute. Le changement radical de la situation financière intervenu en 1991, d'un déficit structurel du compte courant à un excédent record qui a permis d'amasser des réserves en devises couvrant plus d'une année d'importations (18 milliards de dollars à la fin de 1994), a moins pour origine des mesures économiques qu'une «rente géopolitique » particulièrement bien exploitée durant la guerre du Golfe. L'effacement de la moitié de la dette extérieure publique de l'Égypte et l'aide occidentale et arabe sans précédent qui l'a accompagné en sont une conséquence directe, même s'ils ont été consentis à condition que le gouvernement entreprenne un programme d'ajustement structurel contrôlé par les institutions financières internationales ${ }^{1}$.

L'enjeu principal de la conjoncture exceptionnellement favorable que l'Égypte traverse depuis la fin de la guerre du Golfe est le passage d'une économie rentière dirigiste à une économie de production internationalisée.

3 L'insuffisance des résultats enregistrés plus de trois ans après le début des réformes a alimenté les critiques sur la fermeté des autorités égyptiennes. A leur décharge, il semble que le fait que personne ne sache vraiment comment fonctionne l'économie compte pour beaucoup dans leurs atermoiements devant certaines réformes, dont elles ne savent pas toujours où elles les mèneraient. En effet, l'intégration des campagnes dans la modernité est si faible que les partenaires économiques internationaux de l'Égypte ont l'habitude de ne considérer comme solvable qu'environ $10 \%$ de la population, soit environ 6 millions de personnes, chiffre qui paraît cependant sous- 
estimé au regard des dernières enquêtes sur la consommation des ménages. C'est déjà entrevoir combien il est difficile de cerner la réalité économique égyptienne selon les indicateurs traditionnels (voir plus loin le tableau 1). La mesure du niveau de vie en fournit une bonne illustration.

\section{Le niveau de vie égyptien}

4 Avec 2440 dollars en 1991, le revenu par habitant en Égypte calculé selon les taux de change en parités de pouvoir d'achat (PPA) est quatre fois plus élevé que le résultat obtenu selon la méthode traditionnellement utilisée dans les annuaires statistiques internationaux. Les taux de change en parités de pouvoir d'achat sont calculés par le Fonds monétaire international dans ses Perspectives de l'économie mondiale de façon à égaliser les niveaux de prix des différents pays exprimés dans une monnaie commune. Leur usage permet de gommer l'impact des fluctuations des monnaies sur l'évaluation des revenus réels des populations. Les comparaisons internationales effectuées selon ces statistiques sont donc plus réalistes que celles qui sont basées sur les chiffres du PIB et du PIB/habitant. Elles ont pour effet de rapprocher les pays à faible niveau de prix de ceux où les prix sont élevés et les pays pauvres très peuplés des pays plus riches moins peuplés, ce qui resserre l'éventail des revenus entre pays en développement et pays industrialisés.

C'est pourquoi le revenu égyptien moyen, habituellement considéré comme le plus bas du bassin méditerranéen (620 dollars en 1991), dépasse en PPA celui du Maroc et se rapproche de celui des autres pays de la région. Le niveau de vie moyen des Israéliens, par exemple, n'est ainsi plus que 5,4 fois supérieur à celui des Égyptiens et non 18,3 fois, comme l'indiquerait une comparaison des PIB/habitant. Le changement conduit à des reconsidérations encore plus spectaculaires au niveau du revenu national, puisqu'on PPA celui de l'Égypte est multiplié par 3,9, passant de 33 à 130 milliards de dollars en 1991, ce qui lui permet de dépasser le produit intérieur brut d'Israël (67 milliards de dollars), dont il est habituellement considéré comme très éloigné. Les statistiques en PPA ont été critiquées parce qu'elles conduisent à minimiser la pauvreté des pays en développement en leur conférant une taille économique disproportionnée par rapport à leur puissance réelle. Ainsi la Chine devient-elle en PPA la troisième puissance économique mondiale, compte tenu du niveau de ses prix intérieurs et de l'importance de sa population, bien que ses faiblesses économiques ne puissent à l'évidence lui conférer ce rang. A l'inverse, ces statistiques continuent d'ignorer le produit du travail informel, que l'on estime en Égypte à environ un tiers du PIB «officiel» et qu'on considère habituellement comme proportionnellement plus important dans les pays en développement que dans les pays industrialisés.

Une autre tentative récente de renouveler les critères de comparaison des pays entre eux se base sur l'indice de développement humain, qui est une mesure synthétique du revenu par habitant au taux de change en PPA, ainsi que du niveau éducatif (taux d'alphabétisation et nombre moyen d'années d'études) et de la situation sanitaire (mesurée par l'espérance de vie à la naissance) de la population. Bien que cet indice utilise les PPA, il place l'Égypte largement au-dessous des autres pays méditerranéens et moyen-orientaux, ce qui montre que la réévaluation de sa taille économique et du niveau de vie de sa population issue des considérations précédentes ne doit pas cacher les handicaps sociaux, qui mettent en exergue son sous-développement relatif dans la 
région. La mesure de l'évasion des capitaux constitue un autre facteur susceptible de tempérer l'image de pays écrasé par la pauvreté souvent accolée à l'Égypte.

\section{L'épargne égyptienne a l'étranger}

7 Selon un document de travail interne de la Banque mondiale ${ }^{2}$, l'épargne privée détenue à l'étranger par les résidents en Égypte s'élevait à 82,6 milliards de dollars en 1991, soit $271 \%$ du PNB de ce pays et près de quatre fois les 21,5 milliards de dollars représentant le service «réel» de sa dette extérieure, c'est-à-dire la somme que l'Égypte devra effectivement régler à ses créditeurs une fois défalquées les réductions de dettes promises. Ce chiffre est extrêmement élevé, comparativement à ceux qu'enregistraient les pays d'Amérique du Sud avant que ne s'y amorce le mouvement de retour des capitaux (plus de six fois supérieur), ainsi que par rapport aux autres États de la région. En Égypte, l'évasion de capitaux a commencé et a atteint son paroxysme durant l'infitah, représentant 14,5\% du PNB de 1975 à 1979, puis a baissé en termes relatifs, mais d'importantes rentrées nettes (6,4\% du PNB) se sont produites en 1990. Les évasions totales de capitaux se sont élevées à 40,5 milliards de dollars de 1970 à 1991 (le stock d'épargne valorisée atteignant quant à lui 82,6 milliards de dollars), soit sensiblement autant que les transferts nets au secteur public, ce qui signifie que si l'épargne égyptienne avait été investie sur place, elle aurait rendu inutile l'aide étrangère à l'Égypte et donc son endettement extérieur.

D'autre part, l'évasion de capitaux a augmenté parallèlement à la baisse du taux d'investissement (investissements/PIB), si bien que c'est au moment où l'économie aurait eu le plus besoin de l'épargne intérieure que cette dernière a fui le pays, d'où le recours aux financements extérieurs. Ceux-ci ont médiocrement été fournis par les investissements directs étrangers, largement concentrés dans le secteur pétrolier, ce qui n'étonne guère, puisque l'épargne privée locale elle-même était peu dirigée vers l'investissement. Par contre, les transferts des émigrés ont représenté à peu près l'équivalent des évasions de capitaux et les ont donc en gros compensées. On peut croire que si les conditions adéquates pour l'investissement local de l'épargne privée avaient été rassemblées, le taux d'investissement aurait augmenté, y compris grâce à une hausse des investissements directs (effet cumulatif), l'endettement externe aurait diminué (en termes relatifs au moins) et l'émigration aurait été moindre grâce aux opportunités d'emplois offertes par ces investissements, qui auraient permis aux Égyptiens de travailler pour leur pays, et non pour la construction d'autres économies. L'un des principaux objectifs des réformes en cours est d'inverser cette tendance afin que l'investissement soit davantage assuré par l'épargne locale que par les transferts de l'étranger.

\section{Une économie rentière}

9 L'économie égyptienne peut être qualifiée de «rentière » aux niveaux externe et interne:

- Les ressources de rente externe, c'est-à-dire celles qui ne proviennent pas d'un travail local, ont compté au cours des dernières années pour les deux tiers des recettes courantes (tableau 1). Les transferts des Égyptiens de l'étranger viennent en tête avec $26,9 \%$ du total, suivis par les transferts publics (ou aide publique, $10,7 \%$ du total), le canal de Suez $(10,4 \%)$ et les 
exportations pétrolières (10\%). Bien qu'en légère baisse depuis 1991, cette rente reste plus élevée en 1992/93 qu'en 1989/90, et, surtout, très importante en valeur absolue puisqu'elle équivaut au tiers du PIB- Le FMI est conscient de la difficulté de modifier cette situation, puisqu'il prévoit que les rentes externes compteront encore pour $62,3 \%$ des recettes courantes en 1997-98 (tableau 2). En 1993/94, ses prévisions sont toutefois apparues trop optimistes, puisque les exportations non pétrolières ont été inférieures du tiers à ce qu'il escomptait, à 1,61 milliard de dollars seulement, ce qui place l'Égypte au niveau d'un pays comme la Syrie, dont le PIB est quatre fois inférieur.

- Les rentes internes engendrées par la structure des prix relatifs et la protection douanière ont entraîné des déséquilibres et des mésallocations de ressources pour les secteurs public et privé. La politique de vérité des prix suivie depuis 1991 a commencé à corriger ces phénomènes, qui ne pourront disparaître qu'à la faveur d'une reprise de l'investissement. La rigueur budgétaire adoptée depuis lors a entraîné une baisse en valeur des investissements publics (dépenses d'équipement de l'État, plus investissements des entreprises publiques), si bien qu'en 1992/93 ils ont pour la première fois été inférieurs aux investissements privés, qui ont représenté $50,2 \%$ du total. Ceux-ci ont progressé au rythme élevé de 15,6\%, supérieur de 4,5 points au taux d'inflation, alors que la hausse de $7 \%$ des investissements totaux en termes courants signifie une baisse réelle de $4,1 \%$. Bien que les conditions de l'investissement privé ne soient pas encore exemptes de distorsions, on peut conclure à une lutte du programme d'ajustement structurel contre les rentes internes qui tend à porter ses fruits. Une fois la reprise de l'investissement privé confirmée, il restera cependant aux autorités à prendre les mesures nécessaires pour que soient redistribués les fruits de la croissance, ce qui ne pourra advenir que via la réconciliation des pouvoirs politiques et économiques, autrement dit si l'État abandonne sa prétention à monopoliser la légitimité.

\section{Le programme d'ajustement structurel}

10 L'accord conclu avec le Fonds monétaire international en mai 1991 et renouvelé en septembre 1993 a engagé l'Égypte dans un programme de libéralisation économique, dont l'application lui garantit un soutien financier international considérable. Près de quatre ans après sa signature et en dépit des importantes mesures adoptées, le retard mis par le gouvernement à mettre en oeuvre des reformes en profondeur, notamment la restructuration du secteur public et la promotion des exportations grâce à la baisse des taux d'intérêt et du cours de la livre, montre qu'il chemine à son gré. C'est la raison pour laquelle le FMI a refusé en juin, puis en décembre 1994, de donner l'aval nécessaire à l'effacement de la troisième et dernière tranche de la réduction de moitié de la dette extérieure publique égyptienne, prévue pour juillet 1994 selon le calendrier fixé en 1991. Cette dernière tranche porte sur $20 \%$ de sa valeur actualisée à cette date, soit environ 4 milliards de dollars.

11 La réussite de l'ajustement structurel dont se targuent les dirigeants égyptiens en mettant en avant ses résultats financiers semble étroitement liée à la conjoncture géopolitique. Succès certes, mais « rentier ». On sait que lorsque les ressources de rente sont élevées, les économies rentières jouissent de forts taux de croissance, qui chutent avec la baisse de la rente: leur bonne santé apparente est fragile. En matière d'ajustement structurel aussi, il peut exister des succès trompeurs. Comment expliquer la contradiction entre la réussite exceptionnelle de l'Égypte depuis 1991 et le fait qu'elle soit en retard sur ses engagements de réforme économique ? Ce retard aurait dû 
tempérer ses succès. En fait, la rente engrangée à la suite de la guerre du Golfe a permis un placement aisé des bons du Trésor, la stabilité de la livre et la fin de l'évasion des capitaux, donc la réussite apparente de l'ajustement, de façon moins douloureuse que des réformes de structure plus profondes. Cette situation a incité le gouvernement à suivre de front deux stratégies contradictoires, ajustement structurel et préservation de la rente, l'application de la première permettant de pérenniser la seconde. Les accords avec le FMI constituent en effet des conditions nécessaires pour bénéficier des remises de dettes prévues (12,3 milliards de dollars) et de nouveaux crédits des bailleurs de fonds ( 2 milliards de dollars/an). La tactique égyptienne consiste à s'y conformer au minimum et le plus tard possible, tout en évitant la rupture. Les « conditionnalités » instaurées par le FMI deviennent ainsi des moyens pour un objectif financier poursuivi lorsque nécessité fait loi.

Paradoxalement, il apparaît donc que les relations de l'Égypte avec le FMI ne changent pas l'orientation rentière de sa politique économique, ce qui va à rencontre de l'ajustement structurel recherché. C'est pourquoi les réformes accomplies jusqu'à présent n'ont pas fondamentalement modifié la nature de l'économie, contrairement à ce qu'escomptait le Fonds. Ce dernier s'inquiète depuis un an de cette évolution, puisqu'on a noté une accentuation des différends avec les autorités égyptiennes, qui ont progressivement été rendus publics: La question pendante à l'heure actuelle est de savoir si le soutien des États-Unis à l'Égypte suffira à assouplir la position du FMI. En effet, c'est en interprétant l'accord américano-égyptien de "partenariat pour la croissance et le développement ", signé en septembre 1994, comme un renouvellement de l'appui américain, que les autorités égyptiennes n'ont pas hésité à refuser certaines des mesures de politique économique préconisées par le Fonds. Vu le retard pris à définir les modalités d'exécution de cet accord, qui jette un doute sur cette interprétation, il n'est pas certain que l'Égypte obtienne gain de cause cette fois-ci.

\section{Conclusion}

II apparait en définitive que l'économie égyptienne ne mérite pas intrinsèquement son image catastrophique. C'est plutôt un gâchis de ses potentialités qui ressort de l'analyse de certaines de ses caractéristiques menée ci-dessus. Pays classé parmi les plus pauvres, l'Égypte est riche de facteurs de production inemployés. Plutôt que d'exporter sa maind'œuvre et ses ressources énergétiques ou de s'évertuer à percevoir des rentes externes, elle pourrait mettre sa force de travail au service du développement de ses ressources naturelles en aménageant le cadre susceptible de promouvoir les investissements nécessaires à cet effet. Les réformes engagées depuis 1991 en concertation avec les institutions financières internationales vont dans cette direction, mais semblent trop partielles - dans les textes et surtout dans leur application - pour parvenir à un tel résultat.

14 Nulle part cette situation n'apparaît aussi clairement que dans le cas du canal de Suez. L'une des principales voies de transit maritime de la planète (17 500 bateaux par an), ce canal a été construit à proximité d'un immense bassin d'emploi - le Delta du Nil - et la majeure partie de la production égyptienne d'hydrocarbures provient du golfe de Suez. Or, l'Égypte se contente de prélever des droits de transit sur des marchandises qui sont transformées ailleurs, alors qu'elle pourrait exploiter là ses facteurs de production $-\mathrm{y}$ compris l'eau acheminée du Nil, mais pour l'agriculture, et l'espace - que beaucoup de 
pays lui envieraient, pour transformer une partie des marchandises en transit. De meilleures conditions pour les investissements pourraient faire de la région du canal un grand pôle industriel. Instabilité politique et stratégie économique inadéquate ont jusqu'à présent empêché un tel développement. Les réaménagements régionaux et internes qui surviennent depuis 1991 pourraient renverser cette situation.

\section{ANNEXES}

Tableau 1 : Les rentes externes dans l'économie égyptienne (1986/87-1992/93. millions \$)

\begin{tabular}{|l|l|l|l|l|l|l|l|}
\hline & $86 / 87$ & $87 / 88$ & $88 / 89$ & $89 / 90$ & $90 / 91$ & $91 / 92$ & $92 / 93$ \\
\hline Canal de Suez & 1.148 & 1.269 & 1.307 & 1,472 & 1.662 & 1.950 & 1.940 \\
\hline Sumed* & 100 & 100 & 100 & 100 & 100 & 100 & 100 \\
\hline Exportations pétrolières & 1.225 & 1.563 & 1.066 & 1.229 & 1.971 & 1.651 & 1.700 \\
\hline Revenus d'investissement & 1.042 & 898 & 1.097 & 1.164 & 1.416 & 1.293 & 1.292 \\
\hline Transferts privés & 3.012 & 3,384 & 3.532 & 3.743 & 3.755 & 5.467 & 5.000 \\
\hline Transferts publics & 974 & 698 & 756 & 1.094 & 4.842 & 1.349 & 1.330 \\
\hline Sous-total : recettes rentières (a) & 7.501 & 7.912 & 7,858 & 8.802 & 13.746 & 11.810 & 11.362 \\
\hline Exportations non pétrolières & 1.360 & 1.675 & 1.631 & 1.915 & 1.916 & 1.985 & 1.758 \\
\hline Autres services & 2,155 & 2.582 & 2.885 & 3.358 & 3,556 & 3.835 & 4.012 \\
\hline Total : recettes courantes (b) & 11.016 & 12.169 & 12.374 & 14.075 & 19.218 & 17.630 & 17.132 \\
\hline (a)/(b) & $68,1 \%$ & $65,0 \%$ & $63,5 \%$ & $62,5 \%$ & $71,5 \%$ & $67.0 \%$ & $66.3 \%$ \\
\hline (a)/PIB & $17,1 \%$ & $28,9 \%$ & $24,5 \%$ & $27,2 \%$ & $40.2 \%$ & $32,9 \%$ & $28.7 \%$ \\
\hline
\end{tabular}

* Selon mes estimations (I'Arab Petroleum Pipeline Co., qui exploite l'oléoduc Suez-Méditerrannée ou Sumed, ne publie pas ses comptes).

Source : FMI (1992/93 : estimations de septembre 1993) ; calculs personnels.

Tableau 2: Les rentes externes dans l'économie égyptienne (prévisions 1993/94-1997/98, millions \$)

\begin{tabular}{|l|l|l|l|l|l|}
\hline & $93 / 94$ & $94 / 95$ & $95 / 96$ & $96 / 97$ & $97 / 98$ \\
\hline
\end{tabular}




\begin{tabular}{|l|l|l|l|l|l|}
\hline Canal de Suez & 2.223 & 2.381 & 2.551 & 2.732 & 2.927 \\
\hline Sumed * & 150 & 150 & 150 & 150 & 150 \\
\hline Exportations pétrolières & 1.701 & 1.770 & 1.779 & 1.907 & 1.943 \\
\hline Revenus d'investissement & 2.258 & 2.730 & 2.920 & 3.086 & 3.249 \\
\hline Transferts privés & 5.096 & 5.300 & 5.512 & 5.732 & 5.962 \\
\hline Transferts publics & 1.225 & 1.197 & 1.174 & 1.161 & 1.149 \\
\hline Sous-tolat : recettes rentières (a) & 12.653 & 13.528 & 14.086 & 14.768 & 15.380 \\
\hline Exportations non pétrolières & 2.362 & 2.611 & 2.909 & 3.209 & 3.433 \\
\hline Autres services & 4.833 & 5.070 & 5.356 & 5.680 & 5.872 \\
\hline Total : recettes courantes (b) & 19.848 & 21.209 & 22.351 & 23.657 & 24.685, \\
\hline (a)/(b) & $63,7 \%$ & $63,8 \%$ & $63,0 \%$ & $62.4 \%$ & $62.3 \%$ \\
\hline$(a) /$ PIB & $31,4 \%$ & $32,6 \%$ & $32,7 \%$ & $32,7 \%$ & $n . d$. \\
\hline
\end{tabular}

*Selon mes estimations. Source : FMI, janvier 1993 ; calculs personnels.

\section{NOTES}

1. Pour une description détaillée de ce programme, qui relève de deux accords conclus entre l'Égypte et le FMI en 1991 et 1993, voir Blin Louis, "Le programme de stabilisation et d'ajustement structurel de l'économie égyptienne ", Égypte/Monde arabe9, $1^{\mathrm{er}}$ trimestre 1992, p. 13-46 et «Le renouvellement de l'accord entre l'Égypte et le Fonds monétaire international », Égypte/Monde arabe 15-16,1993, p. 297-321.

2. Diwan I, Underwood J., Squire L, External Finance in the Middle East: Trends and Prospects, Washington, the World Bank, Middle East and North Africa Discussion Paper Series, 10, December 1993, $70 \mathrm{p}$.

\section{INDEX}

Mots-clés : économie 